\title{
Theory of Ferromagnetism in Doped Excitonic Condensates
}

\author{
E. Bascones, A. A. Burkov, and A. H. MacDonald \\ Department of Physics, University of Texas at Austin, Austin, Texas 78712
}

(November 21, 2018)

\begin{abstract}
Nesting in a semimetal can lead to an excitonic insulator state with spontaneous coherence between conduction and valence bands and a gap for charged excitations. In this paper we present a theory of the ferromagnetic state that occurs when the density of electrons in the conduction band and holes in the valence band differ. We find an unexpectedly rich doping-field phase diagram and an unusual collective excitation spectrum that includes two gapless collective modes. We predict regions of doping and external field in which phase-separated condensates of electrons and holes with parallel spins and opposing spins coexist.
\end{abstract}

71.35.Lk,73.43.f,73.43.Lp,71.10.Hf,75.30.Kz

It has long been recognized [1,2] that a semimetal or small-gap semiconductor could undergo a phase transition to a state with spontaneous coherence between conduction and valence bands and a gap for charged excitations. When described in terms of electrons in the conduction band and holes in the valence band, this state can be regarded as an electron-hole pair (exciton) condensate, and its mean-field theory description is very similar to the BCS mean-field theory of a superconductor. Excitonic insulator states are expected to occur only if conduction and valence bands are adequately nested.

The excitonic insulator state is evidently rare, most likely [3] because accurate nesting is uncommon and because electron and hole densities are usually not equal. The most compelling example of excitonic-insulator behavior to date is that demonstrated by bilayer quantum Hall systems [4], where a charge gap develops in a state with spontaneous coherence [5] between electrons in different layers. In this paper we consider doped excitonic insulators, i.e. systems in which the conduction-band electron density and valence-band hole density differ. Doping is unfavorable for electron-hole pairing, causing the excitonic insulator to evolve first into a metallic excitonic ferromagnet [6], and finally into a paramagnetic normal metal state. In this paper we address properties of the intermediate ferromagnetic state.

This work is motivated in part by the recent discovery of weak ferromagnetism in lightly-doped divalent hexaborides [7]. These materials are ferromagnetic in spite of the absence of $d$ - or $f$ - partially filled orbitals, and have very high Curie temperatures $T_{c}>600 \mathrm{~K}$ that depend strongly on carrier concentration. Early electronic structure calculations had predicted that these materials would be semimetals or small gap semiconductors [8], leading Zhitomirsky et al [9] to propose excitonic pairing as the source of their ferromagnetism, renewing interest in this unusual magnetic state [10 16]. Uncertainty has been generated, however, by recent experiments 17] which appear to imply that these materials are semiconducting with $\mathrm{a} \sim 1 \mathrm{eV}$ gap, in agreement with more re- cent GW approximation electronic structure calculations [18], casting doubt on the excitonic insulator picture. In this Letter, we present an analysis of the collective excitation spectrum, and doping-chemical potential phase diagram of excitonic insulator ferromagnets, establishing features which could be used to convincingly identify these states on purely experimental grounds. In particular, we demonstrate that doped excitonic condensates are unusual ferromagnets with a characteristic multi-branch collective mode structure.

We start by considering a model Hamiltonian $\mathcal{H}=$ $\mathcal{H}_{0}+\mathcal{H}_{I}$ where $\mathcal{H}_{0}$ includes both conduction and valence band energies and $\mathcal{H}_{I}$ includes all interactions.

$$
\mathcal{H}_{0}=\sum_{\mathbf{k} a \sigma \sigma^{\prime}}\left[\left(\epsilon_{\mathbf{k} a}-\mu\right) \delta_{\sigma, \sigma^{\prime}}-\mathbf{h} \vec{\tau}_{\sigma, \sigma^{\prime}}\right] c_{\mathbf{k} a \sigma}^{\dagger} c_{\mathbf{k} a \sigma^{\prime}}
$$

Here $\mathbf{k}$ denotes the quasimomentum, $\sigma$ the spin, $a=c, v$ is a band label, $\mu$ is the chemical potential and $\mathbf{h}=$ $\frac{1}{2} g \mu_{B} \mathbf{H}$ here $\mathbf{H}$ is an external magnetic field. We start by including only long-range Coulomb interactions, which leads to a model with interaction vertices that conserve band labels and spins:

$$
\mathcal{H}_{I}=\frac{1}{2 \Omega} \sum_{\mathbf{k} \mathbf{k}^{\prime} \mathbf{q} \sigma \sigma^{\prime}} \sum_{a a^{\prime}} V(\mathbf{q}) c_{\mathbf{k}+\mathbf{q} a \sigma}^{\dagger} c_{\mathbf{k}^{\prime}-\mathbf{q} a^{\prime} \sigma^{\prime}}^{\dagger} c_{\mathbf{k}^{\prime} a^{\prime} \sigma^{\prime}} c_{\mathbf{k} a \sigma}
$$

where $\Omega$ is the crystal volume. This interaction model is known as the dominant term approximation [2], and is accurate for low-carrier densities and weak Coulomb interactions. In this model charge and spin are conserved separately in each band, leading to a $S U(2) \times S U(2)$ symmetry [10] that will figure prominently in our discussion.

For simplicity we consider the case of delta-function interactions, $V(\mathbf{q})=V_{0}$, and of isotropic, parabolic valence and conduction bands that are perfectly nested in the absence of doping:

$$
\epsilon_{\mathbf{k} c}=-\epsilon_{\mathbf{k} v}=\frac{\mathbf{k}^{2}}{2 m}-E_{G} .
$$

Here $E_{G}>0$, the case we consider, and $E_{G}<0$ imply semimetallic and semiconducting behavior, respectively. 
Our results for ground states and excitations are based on unrestricted Hartree-Fock and time-dependent Hartree-Fock approximations respectively. Excitonic condensation is driven by interband exchange terms that favor spontaneous phase coherence. The intraband exchange self-energy has a spin-independent contribution which we absorb into $E_{G}$ and an important spindependent contribution which has not always been accounted for in previous work, but plays an essential role in stabilizing the ferromagnetic state 10.

Dropping the direct Hartree interaction which plays no role for homogeneous states, the mean-field Hamiltonian is

$$
\begin{aligned}
\mathcal{H}_{M F} & =\sum_{\mathbf{k} a \sigma \sigma^{\prime}}\left[\left(\epsilon_{\mathbf{k} a \sigma}-\mu\right) \delta_{\sigma \sigma^{\prime}}-\left(\mathbf{h}_{a}+\mathbf{h}\right) \vec{\tau}_{\sigma \sigma^{\prime}}\right] c_{\mathbf{k} a \sigma}^{\dagger} c_{\mathbf{k} a \sigma^{\prime}} \\
& -\sum_{\mathbf{k} \sigma \sigma^{\prime}}\left[\left(\Delta_{s} \delta_{\sigma \sigma^{\prime}}+\vec{\Delta}_{t} \vec{\tau}_{\sigma \sigma^{\prime}}\right) c_{\mathbf{k} c \sigma}^{\dagger} c_{\mathbf{k} v \sigma^{\prime}}+\text { h.c. }\right]
\end{aligned}
$$

Here

$$
\mathbf{h}_{a}=\frac{V_{0}}{\Omega} \mathbf{m}_{a}=\frac{V_{0}}{2 \Omega} \sum_{\mathbf{k} \sigma \sigma^{\prime}} \rho_{a a}^{\sigma \sigma^{\prime}}(\mathbf{k}) \vec{\tau}_{\sigma^{\prime} \sigma}
$$

is the mean-field intraband exchange spin-splitting field (proportional to the band magnetization $\mathbf{m}_{a}$ ),

$$
\begin{aligned}
& \Delta_{s}=\frac{V_{0}}{2 \Omega} \sum_{\mathbf{k} \sigma \sigma^{\prime}} \rho_{c v}^{\sigma \sigma^{\prime}}(\mathbf{k}) \delta_{\sigma \sigma^{\prime}} \\
& \vec{\Delta}_{t}=\frac{V_{0}}{2 \Omega} \sum_{\mathbf{k} \sigma \sigma^{\prime}} \rho_{c v}^{\sigma \sigma^{\prime}}(\mathbf{k}) \vec{\tau}_{\sigma^{\prime} \sigma}
\end{aligned}
$$

are the singlet and triplet excitonic condensate order parameters, and

$$
\rho_{a a^{\prime}}^{\sigma \sigma^{\prime}}(\mathbf{k})=\left\langle c_{\mathbf{k} a^{\prime} \sigma^{\prime}}^{\dagger} c_{\mathbf{k} a \sigma}\right\rangle
$$

is the Hartree-Fock density matrix.

We have solved these equations for a range of chemical potentials and external fields, allowing for density matrices that have spontaneous coherence between conduction and valence bands and either spontaneous or induced spin-polarization in conduction and valence bands. Our results are summarized in Fig. 1. There are in general several families of solutions, a normal state solution (N) in which no symmetries are broken, a ferromagnetic excitonic solution in which the excitonic condensate involves only conduction band electrons and valence band holes with particular spin orientations (see the inset in Fig. 1a)), and the excitonic insulator (EI) state in which both spin orientations participate in the excitonic condensate. Invariance of the Hamiltonian under independent spinrotations of conduction and valence band systems in the absence of an external magnetic field is key to understanding the phase diagram. For zero chemical potential and magnetic field a family [2] of symmetry-related EI states is lowest in energy. For $h=0$, a family of ferromagnetic states forms the ground state over a finite range of $\mu \neq 0$. This family includes states with different spin orientations for condensed conduction and valence band particles, and states with different total magnetization magnitude. A weak external magnetic field selects from this family the state which has the largest magnetization magnitude aligned with the field direction. For $\mu \geq 0$ this is always a state in which spin down valence holes are condensed. For large $\mu$, where the largest contribution to the conduction band magnetization comes from uncondensed electrons, it follows that the collinear (COL) state which has coherence between parallel spin conduction and valence band electrons, is the ground state. For small $\mu$, on the other hand, the non-collinear state (NC) is selected by a field. Both COL and NC ferromagnetic excitonic states compete with the EI state in which all spin orientations are paired, and with the $\mathrm{N}$ state, with the four different states separated by first order phase transitions. Both magnetic and pairing order parameters depend on both magnetic field and chemical potential, varying continuously within a phase and discontinuously at a phase boundary. In region EI2 only spin down valence band electrons and spin up conduction band holes are condensed. Unlike the NC state, this phase is insulating.

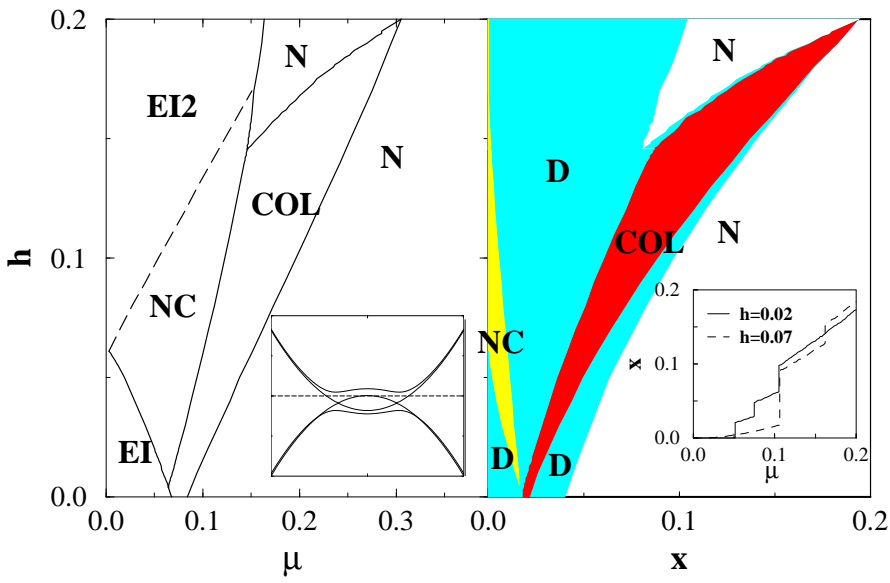

FIG. 1. Phase diagram in the presence of a field as a function of chemical potential $\mu$ a) or doping $x$ b) and field $h$ for $E_{g}=0.1$ and $V_{0}=1.1$. Total electronic density is given by $n_{0}(1+x)$. Energy is measured in units of the band width $\pi^{2} /(2 m)=1$. The adjacent pure phases occur as phase separated domains in the regions labelled $\mathrm{D}$ in $\mathrm{b}$ ). The inset in a) shows a sketch of the Hartree-Fock quasiparticle bands in the ferromagnetic state. The inset in b) shows the dependence of doping on chemical potential.

The slopes of the first order phase boundary lines are related to density and magnetization discontinuities by the following Clapeyron equation

$$
\frac{d h}{d \mu}=-\frac{n_{0} \Delta x}{\Delta M}
$$


Here $x$ is the dimensionless quantity used to measure density and $n_{0}$ is the density in the absence of doping. As shown in the inset of Fig.1b) $x$ always increases upon crossing these phase boundaries in the direction of increasing chemical potential. Positive slopes for these phase boundary lines correspond therefore to a magnetization that decreases when crossing the phase boundary in the same direction. Inside each phase, density increases monotonically with chemical potential. It follows that as a function of density, the phase diagram consists of pure phase regions interspersed with regions of phase separation. The regime consisting of phase separated COL and NC states, which have different magnetizations and excitonic pairing of quite different character, is particulary unusual. Note also the reentrant normal state, predicted earlier [11, induced in the COL by field at large $x$.

Given the unusual nature of these ferromagnets, we can expect that their collective mode structure is unusual. We have evaluated the dynamic linear response in a time-dependent Hartree-Fock approximation [20]. We discuss here only spin-wave collective excitations, those that involve tilts of the magnetization orientation and are therefore off-diagonal in spin-indices when the valence and conduction band mean-field magnetization directions are chosen as quantization axes.

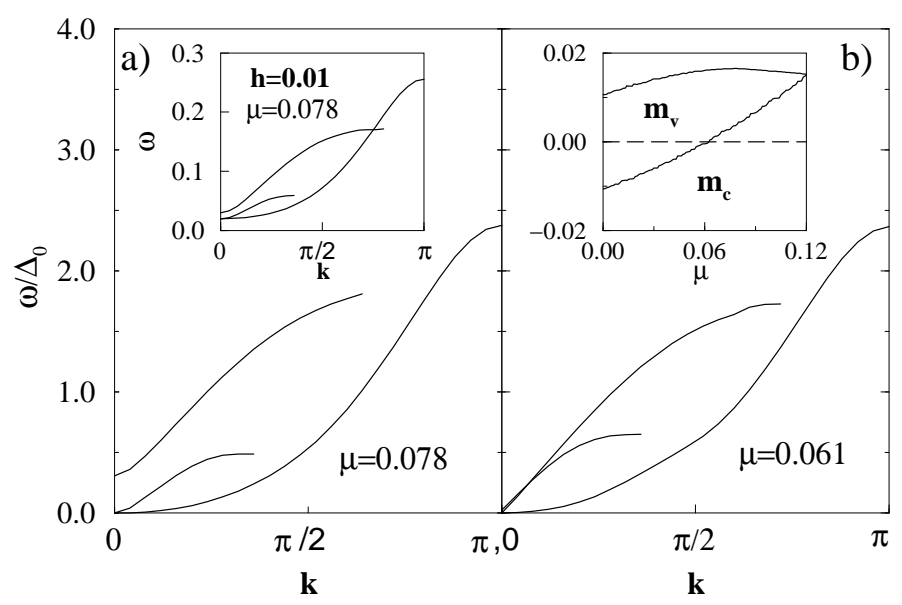

FIG. 2. Spin wave energies in units of the $h=0, \mu=0$ excitonic insulator order parameter $\Delta_{0}=0.1$ for the COL pure state. We measure the momentum in units of the lattice parameter. These results were obtained for $E_{g}=0.1$ and $V_{0}=1.1$, in units of the bandwidth. The main figures show zero field results. for two different values of the chemical potential corresponding to positive and zero conduction band magnetizations respectively The inset in b) shows the dependence of the COL state valence and conduction band magnetizations on $\mu$. Magnetization is measured in units of $n_{0}$. The inset in a) shows the collective excitation spectrum at finite field.

Our results for the spin-wave collective modes $E(q)$ of pure phase excitonic ferromagnets are summarized in
Fig. 2. For $h=0$, there are two gapless collective modes with quadratic dispersion, corresponding to the independent conduction and valence band broken spin rotational symmetries, and an additional soft but gapped collective mode. An interesting feature occurs in the doping dependence of this excitation spectrum. At an isolated value of $\mu$ the spectrum consists of one quadratically dispersing mode and two linearly dispersing modes. To understand the origin of this odd behavior, it is necessary to understand how the separate conduction and valence band contributions to the magnetization vary with doping. Consider, for example, the collinear state. For the electron doping case illustrated here there are no uncondensed valence band holes. Uncondensed conduction band electrons, on the other hand, make a contribution to the magnetization opposite to that of the condensed conduction band electrons, yielding a net magnetization. At some point, the overall magnetization from the conduction band vanishes. This is point at which the collective excitation spectrum is anomalous, and incidentally also the same point, at which COL and NC states have the same magnetization. Apparently the conduction band excitation spectrum at this point is much like that of an antiferromagnet, with condensed and uncondensed band electrons producing opposing magnetization contributions.

In the presence of an external magnetic field our calculations show that there are two Larmour collective modes at energy $E=2 h=g \mu_{B} H$. At first sight the fact that conduction and valence collective modes both occur at this energy appears to suggest that their moments interact only with the external magnetic field and not with each other. If this were the case, however, each contribution to the magnetization would simply align with the field and there would be no energy barrier between collinear and non-collinear states. Furthermore, it is clear from our microscopic calculations that conduction and valence bands are coupled by the combined influences of excitonic pairing and Fermi statistics. This surprising in-field collective mode behavior can be understood by considering the dependence of energy on the orientations of the conduction and valence band spins that participate in the pairing. In Fig. 3 we plot, for both collinear and non-collinear states, energies obtained from self-consistent solutions of the mean-field equations for pairing between down spin valence band holes and conduction band spins that have polar orientation angle $\theta_{c}$ with respect to the magnetic field. We see in Fig. 3 that there is indeed a non-trivial dependence of the energy on $\theta_{c}$ with the global minimum occurring at $\theta_{c}=\pi$ in the COL region of the phase diagram and at $\theta_{c}=0$ in the $\mathrm{NC}$ region of the phase diagram. Why then do the conduction and valence band collective magnetization fluctuations not reflect this dependence on their relative orientations? The answer again can be found in the symmetry of Hamiltonian. In a magnetic field, the 
energy of the system is still invariant under independent rotations of conduction and valence band magnetizations around the field axis. Spin-wave collective excitations are related to the leading second order changes in collective energy when magnetization is tilted away from the field direction. Since we are allowed to include only contributions that are invariant under separate conduction and valence band spin-rotations, the quadratic coupling term $\hat{m}_{\perp, c} \cdot \hat{m}_{\perp, v}$, is not allowed. Higher order terms in the expansion of the magnetization, for example ones that go like $\left(\hat{m}_{\perp, c} \cdot \hat{m}_{\perp, c}\right)\left(\hat{m}_{\perp, v} \cdot \hat{m}_{\perp, v}\right)$, and cause interactions between spin-waves, are symmetry allowed.

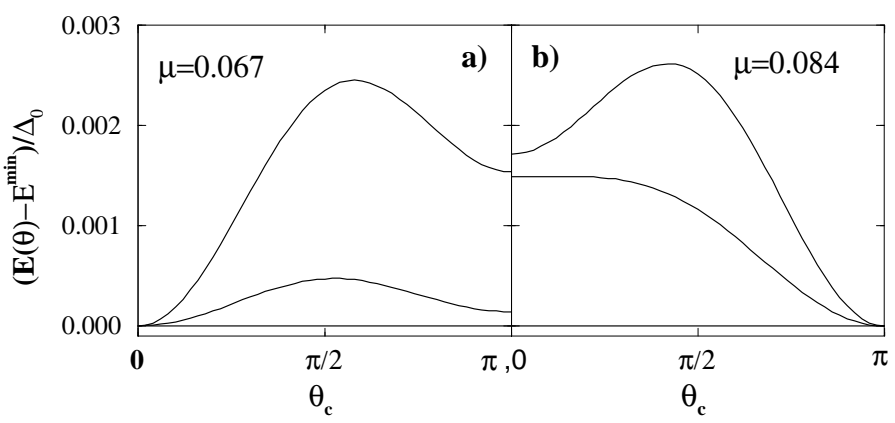

FIG. 3. Dependence of the energy on the angle between the spin of the condensed conduction band and the magnetic field for $\mu=0.067$ and $\mu=0.084$ in which the ground state is at finite field in the NC and COL states respectively, and $\theta_{v}=\pi$. From bottom to top $h=0.0,0.01,0.02$.

It is interesting to speculate on differences between the role of fluctuations in EI ferromagnets, compared to their role in other more familiar magnetic states. At low-temperatures, the Bloch $T^{3 / 2} M(T)$ law should still apply, despite the existence of several low-energy spinwave branches. At higher temperatures, excitonic and magnetic order parameters vanish simultaneously at the mean-field level. We expect that mean-field theory critical temperature estimates should be reliable in the weakcoupling limit $V_{0} \ll E_{g}$.

In closing, we remark that in any real excitonic insulator ferromagnet, corrections to the dominant term approximation would partially alter the picture outlined here. The largest corrections would likely come from interband interaction terms of the form $\delta V_{0} c_{\mathbf{k}+\mathbf{q} c \sigma}^{\dagger} c_{\mathbf{k}^{\prime}-\mathbf{q} v \sigma^{\prime}}^{\dagger} c_{\mathbf{k}^{\prime} c \sigma^{\prime}} c_{\mathbf{k} v \sigma}$. This term will produces corrections to the Hartree-Fock energy of the form $\tilde{E}^{H F} \propto 2\left|\Delta_{s}\right|^{2}-\mathbf{m}_{c} \mathbf{m}_{v}$ We find that the first term is always larger in magnitude, thus favoring triplet pairing and NC orientation in the ferromagnetic phase. The second term favors parallel conduction and valence band magnetizations at $h=0$, coupling the two Goldstone modes and gapping one of them. The resulting $h-\mu$ phase diagram will depend on the competition of magnetic energy and correction terms. In the weak coupling limit $\Delta_{0} \ll\left|E_{g}\right|$, these terms are expected to be negligible and the phase diagram qualitatively unchanged.
We gratefully acknowledge discussions with L. Balents, J. Fernández-Rossier, Z. Fisk, T.M. Rice and M.E. Zhitomirsky. This work has been supported by nsfdmr0115947 and the Welch Foundation.

[1] L.V. Keldysh and Yu. V. Kopaev, Sov. Phys. Solid State 6, 2219 (1965). J. des Cloizeaux, J. Phys. Chem. Solids 26, 259 (1965).

[2] B.I. Halperin and T.M. Rice, Solid State Physics, 21, F. Seitz, D. Turnbull and H. Ehrenreich, eds., Academic Press, New York, 1968.

[3] S. Conti, G. Vignale and A.H. MacDonald, Phys. Rev. B 57, R6846 (1997)

[4] I.B. Spielman, J.P. Eisenstein, L.N. Pfeiffer, and K.W. West, Phys. Rev. Lett. 84, 5808 (2000);ibid 87,36803 (2001).

[5] K. Moon, H. Mori, K. Yand, S.M. Girvin, A.H. MacDonald, L. Zheng, D. Yoshioka and S.-C. Zhang, Phys. Rev. B 51, 5138 (1995).

[6] B.A. Volkov, Yu. V. Kopaev and A.I. Rusinov., Sov. Phys. JETP 41, 952 (1975). B.A. Volkov, A.I. Rusinov and R. Kh. Timerov., Sov. Phys. JETP 43, 589 (1976)

[7] D.P. Young, D. Hall, M.E. Torelli, Z. Fisk, J.L. Sarao, J.D. Thompson, H.-R. Ott, S. B. Oseeroff, R.G. Goodrich and R. Zysler, Nature 397, 412 (1999).

[8] A. Hasegawa and A. Yanase, J. Phys. C 12, 5431 (1979). S. Massidda, A. Continenza, T.M. de Pascale and R. Monnier, Z. Phys. B 102, 83 (1997).

[9] M.E. Zhitomirsky, T.M. Rice and V.I. Anisimov, Nature 402, 251 (1999).

[10] L. Balents and C.M. Varma, Phys. Rev. Lett. 84, 1264 (2000).M.Y. Veillette and L. Balents, Phys. Rev. B 65, 14428 (2002).

[11] V. Barzykin and L.P. Gor'kov, Phys. Rev. Lett. 84, 2207 (2000).

[12] M.E. Zhitomirsky and T.M. Rice, Phys. Rev. B 62, 1492 (2000).

[13] L. Balents, Phys. Rev. B 62, 2346 (2000).

[14] T. Ichinomiya, Phys. Rev. B 63, 5113 (2001).

[15] T.A. Gloor, M.E. Zhitomirsky and T.M. Rice, cond-mat 0010344

[16] S. Murakami, R. Shindou, N. Nagaosa and A.S. Mishchenko, cond-mat 0107215.

[17] J.D. Denlinger, J.A. Clack, J.W. Allen, G.-H. Gweon, D.M. Poirier, C.G. Olson, J.L. Sarrao, Z. Fisk, cond-mat 0009022, ibid 0107429

[18] H.J. Tromp, P.van Gelderen, P.J. Kelly, G. Brocks and P.A. Bobbert, Phys. Rev. Lett 87, 16401 (2001)

[19] Some past work has incorrectly predicted energy differences between states that are related by transformations that are in the symmetry group of the Hamiltonian.

[20] In the EI case we recover the acoustic modes previously predicted in A.N. Kozlov and L.A. Maksimov, Sov. Phys. JETP 22, 889 (1966) and D. Jérome, T. M. Rice and W. Kohn, Phys. Rev. 158, 462 (1967). Due to the 
$S U(2) \times S U(2)$ symmetry of the dominant term approximation, this branch is 4-degenerate. Two of these modes corresponds to the phonons of the condensates while the character of the other two depends on the properties of the selected ground state, e.g. the magnons of the spin density wave in the triplet state. 